\title{
In situ RHAMM protein expression in acute myeloid leukemia blasts suggests poor overall survival
}

\author{
Alexandar Tzankov • Ulrich Strasser • Stephan Dirnhofer • Thomas Menter • \\ Caroline Arber • Martine Jotterand • Alicia Rovo • Andre Tichelli • \\ Reinhard Stauder • Ursula Günthert
}

Received: 17 September 2010 / Accepted: 10 January 2011 /Published online: 28 January 2011

(C) Springer-Verlag 2011

\begin{abstract}
Treatment options for patients with high-risk acute myeloid leukemia (AML) include high-dose chemotherapy regimens in combination with allogeneic hematopoietic stem cell transplantation, which takes advantage of the donor Tcell-mediated graft-versus-leukemia effect. Together with beneficial responses observed in assays targeted at leukemia-associated antigens (LAA), this encouraged research on cancer vaccines and adoptive cellular therapies in AML. The receptor for hyaluronic acid-mediated motility (RHAMM, CD168) was identified as one of the most promising LAA in AML. Thus far, little is known about in situ expression in leukemic bone marrow blasts or the prognostic role of RHAMM and its interaction partners in AML. We immunohistochemically analyzed the expression
\end{abstract}

Alexandar Tzankov and Ulrich Strasser equal contribution.

A. Tzankov $(\triangle) \cdot$ U. Strasser $\cdot$ S. Dirnhofer $\cdot$ T. Menter •

U. Günthert

Institute of Pathology, University Hospital Basel,

Schönbeinstr. 40,

4031 Basel, Switzerland

e-mail: atzankov@uhbs.ch

U. Strasser

Institute of Pathology, Medical University of Innsbruck,

Innsbruck, Austria

C. Arber $\cdot$ A. Rovo $\cdot$ A. Tichelli

Hematology, University Hospital Basel,

Basel, Switzerland

M. Jotterand

Service de génétique médicale, CHUV,

Lausanne, Switzerland

R. Stauder

Department of Internal Medicine V (Hematology and Oncology),

Medical University of Innsbruck,

Innsbruck, Austria and prognostic significance of RHAMM on trephine bone marrow biopsies from 71 AML cases that had been evaluated for cytogenetics and presence of FLT3-internal tandem duplications and NPM1 mutations. Fifty-five patients $(77 \%)$ were treated with curative intent, while $16(23 \%)$ received the most appropriate supportive care. Twenty of 71 (28\%) AML cases were considered RHAMM+. Receiver operating characteristic curves showed significant discriminatory power considering overall survival $(\mathrm{OS})$ in AML patients treated curatively for RHAMM $(p=0.015)$. Multivariable analysis revealed that expression of RHAMM in $>5 \%$ of leukemic blasts identifies a subgroup of curatively treated cases with adverse OS independent of failures to achieve complete remission. RHAMM not only represents a promising LAA with specific T-cell responses in AML but, if assessed in situ on blasts, also a probable prognostic factor.

Keywords AML $\cdot$ RHAMM $\cdot$ CD44 $\cdot$ Prognosis

\section{Introduction}

Acute myeloid leukemias (AML) are neoplastic hematopoietic stem cell disorders characterized by maturation arrest, uncontrolled proliferation, and resistance to apoptosis $[1,2]$. Decision making for treatment of AML patients is risk adapted to the World Health Organization (WHO) defined "clinico-pathologic-genetic" entities [3, 4] and further depends on the patient's health status and age. Treatment options range from best supportive care to intensive multiagent chemotherapy alone or high-dose regimens in combination with, primarily, allogeneic hematopoietic stem cell transplantation (allo-HSCT) [5]. In the latter, the graft-versus-leukemia effect suggests that leukemiaspecific $\mathrm{T}$ lymphocytes play a major role in attacking and 
eliminating leukemic cells, thus supporting long-term remission. Along with several observed immunological and clinical responses using assays targeted at leukemiaassociated antigens, these observations encouraged research on cancer vaccines and adoptive cellular therapies in AML [6-8]. The receptor for hyaluronic acid-mediated motility (RHAMM, CD168) was identified as one of the most promising leukemia-associated antigens in AML [7, 9-13]. RHAMM has several known functions that vary with respect to its different interaction partners. Interaction with CD44 in the presence of hyaluronic acid plays a role in cellular motility, wound healing, and modification of the Rat sarcoma (Ras)-signaling cascade [14-17]. Through interactions with signaling cascades related to extracellular signalregulated protein kinase $1 / 2$ (ERK1/2), breast cancer 1 (BRCA1), and BRCA1-associated RING domain 1 (BARD1), RHAMM, alone or in complex with CD44, osteopontin (OPN), and integrins, can promote cancer cell invasion and is essential for tumorigenicity, most likely by oncogenic inhibition of apoptosis [13-15, 18-23]. Furthermore, intracellular RHAMM is expressed in a cell cycledependent manner and helps maintaining genomic stability, thus acting as a tumor suppressor [21, 24]. Finally, as a surface molecule on tumor cells, RHAMM has been shown to play an important role in humoral anti-tumor responses $[11,25]$. RHAMM has been identified as an important prognostic factor of significance in carcinomas and lymphomas, and also in leukemias [10, 25-33]. A study of Greiner et al. suggested a probable but statistically not significant favorable prognostic impact of elevated RHAMM mRNA expression in AML [7].

Along with RHAMM, CD44, a transmembrane glycoprotein, is also a receptor for hyaluronic acid. CD44 can interact with various molecules, like OPN, matrix metalloproteinases, CD95, and also with extracellular RHAMM to promote invasion, angiogenesis, inflammation, and apoptotic resistance $[19,33,34]$. Like RHAMM, CD44 is involved in signaling pathways including cellular sarcoma (c-Src) family kinases and Ras-like family GTPases [34]. CD44 arouse attention in AML research when a monoclonal antibody against this molecule eradicated AML stem cells [35].

Despite the potentially promising observations on RHAMM in AML, little is known about its in situ expression on bone marrow blasts or its prognostic significance. In addition, there are no data on the expression of its interaction partners, OPN, CD44 and signal transducers, and activators of transcription (STAT), or about its influence on the rate of spontaneous apoptosis in AML. Therefore, we performed immunohistochemical analyses on bone marrow trephine biopsies from a cohort of $71 \mathrm{AML}$ cases to determine the expression and prognostic importance of RHAMM and interacting molecules.

\section{Patients, materials, and methods}

\section{Patients}

A retrospective cohort of 71 patients with AML was analyzed. All newly diagnosed patients with AML between 2005 and 2007 at the University Hospital Basel, who had been fully evaluated for cytogenetics and molecular markers, including the presence of internal tandem duplications of FMS-like tyrosine kinase 3 (FLT3-ITD) and, in cases with normal cytogenetics, for Nucleophosmin 1 (NPM1) mutations, were included in this study if paraffin blocks of the bone marrow trephine biopsies were adequately preserved for immunohistochemical analyses. All cases were reclassified according to WHO criteria [4]. Clinical and follow-up data were obtained by reviewing the charts. Retrieval of tissue and clinical data were performed according to data safety laws.

Of the 71 patients included in this study, 33 were male (mean age 58, median age 57, range 26-95) and 38 female (mean age 56, median 59, range 20-89). Therapy was risk adapted to the "clinico-pathologic-genetic" AML entity and to the patient's health status according to the protocols of the Dutch-Belgian Cooperative Trial Group for Hematology and Oncology (HOVON, http://www. hovon.nl/trials/trials-by-type/aml.html) at the time of diagnosis. The distribution of cases according to the WHO classification is presented in Table 1. Responses to treatment were defined as published by the International Working Group for Diagnosis, Standardization of Response Criteria, Treatment Outcomes, and Reporting Standards for Therapeutic Trials in AML [36].

Cytogenetic analysis was performed at the Centre Hospitalier Universitaire Vaudois in Lausanne (Unité cytogénétique du cancer). Karyotypes were described according to the International System for Human Cytogenetic Nomenclature [37]. Cases were grouped according to cytogenetics into three risk groups as suggested by Grimwade [38]. FLT3-ITD and NMP1 mutation analysis was performed as described [39].

Immunohistochemical staining

Paraffin blocks of the 71 bone marrow trephine biopsies were processed in a standardized manner for immunohistochemistry. Eight cases with reactive bone marrow changes (non-hemolytic anemia and thrombocytopenia) served as controls. Table 2 lists the primary antibodies used as well as the incubation and pre-treatment conditions. Visualization of bound secondary antibodies was done by avidin-biotin-peroxidase techniques using either diaminobenzidine (DAB) or aminoethylcarbazole (AEC) as chromogens. Since the staining intensity varied between cases 
[40, 41], only the relative proportion (percentage) of positively staining tumor cells was quantified. To study reproducibility of stainings, a second observer reassessed $20 \%$ of cases.

\section{Statistics}

Statistical analyses were performed using SPSS version 15.0 (SPSS, Chicago, IL). Incomplete data were not excluded from the tests. The degree of agreement between the observers for the quantitative immunohistochemical values was evaluated by intra/interclass correlation coefficients using the Cronbach's alpha analysis. The Spearman's test was used to analyze relationships between the markers. Only correlation coefficients $>0.300$ or $<-0.300$ were further considered. The Fisher's exact test was used to analyze differences in distribution of case numbers between groups. Mann-Whitney or Kruskal-Wallis tests were applied as appropriate to assess differences of means between groups. The prognostic performance of the variables and determination of optimal cut-off values was established by receiver operating characteristic (ROC curves plotting sensitivity versus 1 -specificity with special consideration of the respective area under the ROC (AUROC)). The optimal cut-off point was calculated using Youden's index $(Y)$, denoting $Y=$ sensitivity + specificity -1 , since this method can be applied to find the optimal cut-off value with the highest sensitivity and specificity when there is no particular requirement on sensitivity and/or specificity [42]. OS was analyzed by the Kaplan-Meier method and compared by the log-rank test in univariable modus and by the Cox' regression analysis in multivariable modus. Only factors that were determined to be of at least trend prognostic significance in the univariable modus $(p<0.1)$ were entered into the multivariable analysis. Statistical significance was defined as $p<0.05$ and corrected for multiple testing, when necessary. Two-sided tests were used throughout.

\section{Results}

Response to therapy and clinical outcomes

The average follow-up time was 16.7 months (median 10; range $0-62$ ). Within this time, 32 patients died. Most of the patients $(n=55)$ were treated with curative intent (chemotherapy alone: $n=25$; intensive chemotherapy followed by autologous HSCT: $n=4$; intensive chemotherapy followed by allo-HSCT: $n=26$ ). Sixteen patients did not qualify for intensive treatment. Five were given hydroxyurea and the others received best supportive care. The mean OS of patients treated without intention to cure was 4.4 months and the median 1.3 months, while the OS of patients treated 
Table 2 Applied antibodies and staining conditions

$A E C$ aminoethylcarbazole, $D A B$ diaminobenzidine, EDTA ethylenediaminetetraacetate, $M W$ microwave oven

\begin{tabular}{llllll}
\hline Antibody & Clone & Source & Dilution & Retrieval & Dye \\
\hline Active caspase3 & AF835 & R\&D & $1: 2,000$ & MW, EDTA pH 8, 100 ${ }^{\circ} \mathrm{C}, 15^{\prime}$ & AEC \\
OPN & OP3N & Novocastra & $1: 50$ & MW, citrate pH 6, 100 $\mathrm{C}, 30^{\prime}$ & AEC \\
panCD44 & IM7.8.1 & ATCC & $1: 100$ & MW, citrate pH 6, 100 C, 30' & AEC \\
RHAMM & 2D6 & Novocastra & $1: 25$ & Bond max, EDTA pH 8, 100 ${ }^{\circ} \mathrm{C}, 20^{\prime}$ & DAB \\
\hline
\end{tabular}

with intention to cure was 45.3 months (mean) and the median was not reached. Of the 55 patients treated with curative intent, 29 achieved a complete remission after induction, 12 a partial remission, while 14 had a resistant disease. Responses to induction therapy did not correlate with leukemia subtype, neither subtypes according to WHO nor with Grimwade's prognostic groups [4, 38], patients' age, regimen composition nor any of the studied phenotypic and genetic markers. Failure to achieve complete remission was a prognostic factor for OS $(p=0.039)$, while patients' age, gender, leukemia subtype were not, yet the KaplanMeier survival curves indicated poorer outcomes for elderly patients and patients with therapy-related AML as well as such with complex karyotypes. These observations were particularly due to the fact that patients treated with curative intent were treated in different ways according to their "clinico-pathologic-genetic" risk and patients being at risk were treated in a more intensive way, which probably evened outcomes (Table 1).

One third of AML express cytoplasmic/membranous RHAMM in approximately $20 \%$ of blasts

Assessment of RHAMM and active caspase-3 expression was highly reproducible (average interobserver correlation coefficient $\alpha=0.92$ and 0.89 , respectively), and that of panCD44 and OPN was good $(\alpha=0.82$ and 0.79 , respectively). Except for 10 AML cases that were not assessable for active caspase-3 quantification, all cases were evaluable by immunohistochemical analysis.

In reactive bone marrows, panCD44 was expressed in erythropoietic precursors, as expected [43], and the same was found with OPN. RHAMM was detectable in single myelo-/ monopoietic precursors and mature mononuclear cells, as formerly shown $[29,44]$. Active caspase-3 stained two to 16 apoptotic bodies per $0.283 \mathrm{~mm}^{2}$, especially in areas rich in erythropoiesis. Qualitative impression of the various antibody staining patterns can be extracted from Fig. 1a and b.

Compared to controls, AML cases showed primarily qualitative staining differences, expressing the respective molecules in different cell populations. PanCD44 was strongly expressed in a mean of $38 \%$ of AML blasts in the 16 positive cases (cut-off score $>5 \%$ ), RHAMM in a mean of $19 \%$ of AML blasts in the 20 positive cases (cut-off score $>5 \%$ ) and OPN in $52 \%$ of AML blasts in the 35 positive cases (cut-off score $>25 \%$ ). The sub-cellular localization of stainable RHAMM was predominantly cytoplasmic. In AML cases with blasts negative for the respective marker, there were few erythropoietic or mononuclear cells remaining, occasionally expressing panCD44, OPN, or/and RHAMM, which served as internal positive control. Active caspase-3 stained 0 to 29 apoptotic bodies per $0.283 \mathrm{~mm}^{2}$, which were morphologically attributable to leukemic blasts. Quantitative immunohistochemical data are shown in Table 1. Qualitative impression of the various antibodies' staining patterns can be extracted from Fig. 1c-f.

Expression of RHAMM in AML correlates with panCD44, pSTAT3, and pSTAT5, and with the spontaneous apoptotic activity

Expression of the analyzed markers was correlated with each other and to known clinical and pathological parameters previously determined on the study collective, such as WHO leukemia subtype, patient age and gender, lack of complete remission, expression of pSTAT1, pSTAT3, and pSTAT5, and presence of FLT3-ITD and NPM1 mutations [39]. RHAMM correlated with expression of panCD44 $(\rho=0.560)$, of pSTAT3 and pSTAT5 $(\rho=0.521$ and 0.347 , respectively) and with spontaneous apoptosis as determined by the expression of active caspase-3 $(\rho=0.321)$. The correlations of RHAMM and panCD44 as well as pSTAT3 and pSTAT5 expression were also observable in AML with recurrent genetic aberrations and AML, not otherwise specified; in addition in AML with normal karyotype and noncomplex abnormalities according to Grimwade [38], presence of FLT3-ITD correlated with failures to achieve complete remission $(\rho=0.504)$. In AML with myelodysplasia-related changes RHAMM correlated with pSTAT1 expression in blasts $(\rho=0.565)$, while this correlation was weaker $(\rho=0.288)$ in the AML collective as a whole. There were no correlations between the studied markers and NPM1 mutation status.

RHAMM is a WHO subtype-, age-, treatment type-, and response to treatment-independent negative prognostic factor in AML

Patients treated with curative intent $(n=55)$ and those receiving best supportive care $(n=16)$ were analyzed 
Fig. 1 Expression of the studied markers. a Expression of panCD44 on erythroid precursors in normal bone marrow. b Expression of RHAMM in a single mononuclear cell in the normal bone marrow. c PanCD44positive AML. d AML case with $20 \%$ RHAMM-positive blasts. e Osteopontin-positive AML. f AML case with increased $(>1 \%)$ spontaneous apoptotic activity as assessed by the expression of active caspase- 3 ; all original magnifications $\times 400$

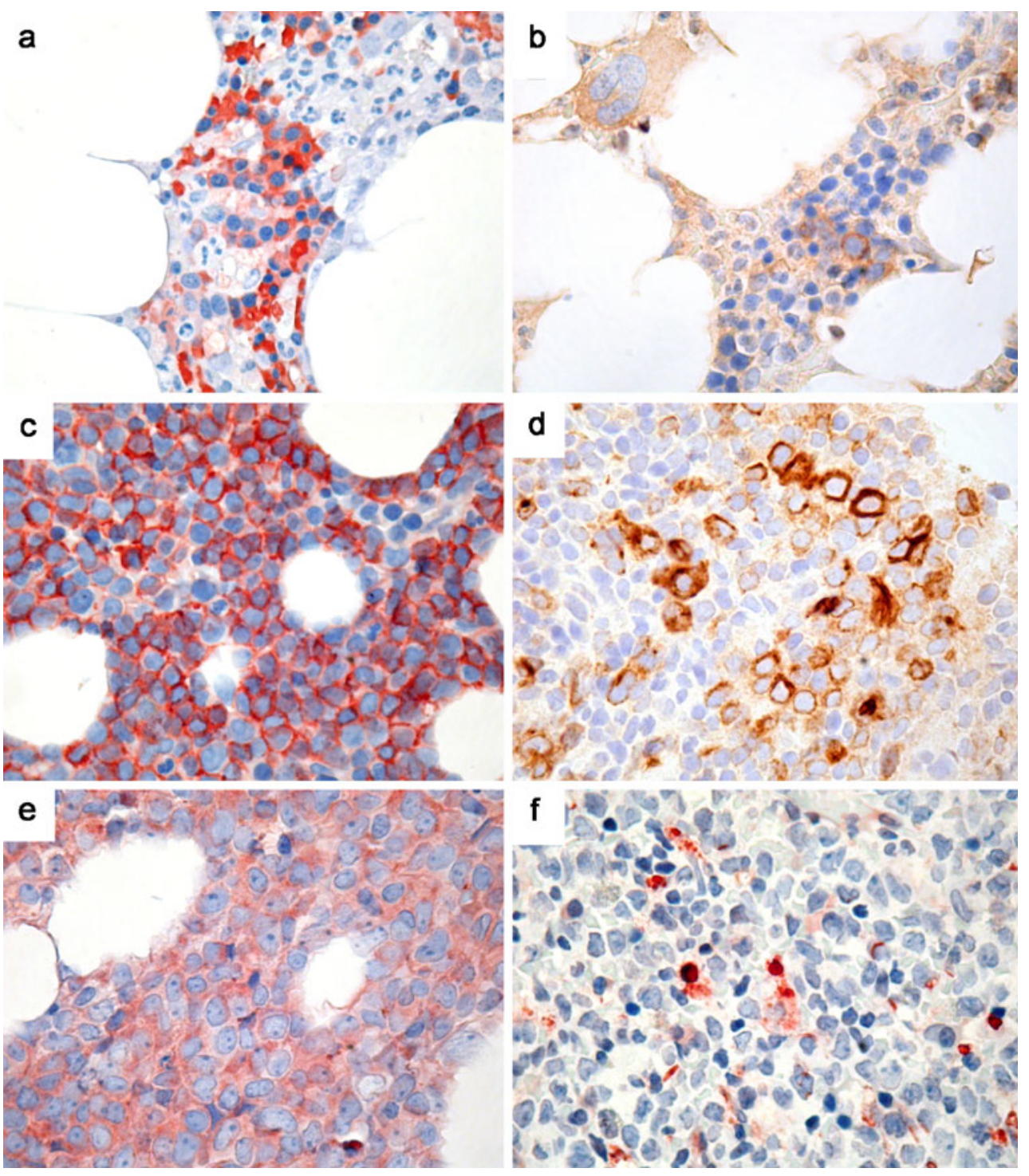

separately to exclude treatment effect bias. Of the markers analyzed, ROC curve-based analysis showed significant discriminatory power for survival of curatively treated AML patients only for RHAMM (AUROC $=0.721, p=0.015$; Fig. 2a). In a further step, this was linked to survival by the Kaplan-Meier method and compared by the log-rank test using the cut-off score suggested by ROC, i.e., $>5 \%$ blasts; the expression of RHAMM showed a significant negative prognostic effect (Fig. 2b) that was particularly evident in patients who failed to achieve complete remission after induction treatment (Fig. 3a and b). Importantly, expression of RHAMM in patients who did or did not achieve complete remission was similar, and the lack of complete remission did not correlate to any of the above-mentioned clinical or pathological parameters. Analyzed with respect to the WHO entities and the Grimwade's cytogenetic prognostic groups [38], the negative prognostic effect of RHAMM was apparent in the Kaplan-Meier curves in AML with recurrent genetic aberrations, in AML, not otherwise specified, in the favorable risk group AML and in AML with normal karyotypes and other noncomplex abnormalities (intermediate risk group) according to Grimwade. Except for AML with normal karyotypes and other noncomplex abnormalities (intermediate risk group), where statistical significance was reached $(p=0.029)$ patient numbers were too low in any of the groups to draw statistical conclusions. Considering the type of curative treatment regimens, this effect remained significant only for patients treated with multiagent chemotherapy $(p=0.031)$, and not with high-dose regimens with HSCT $(p=0.119)$. In a multivariable model, expression of RHAMM turned out to be a negative prognostic factor independent of failure to achieve complete remission in curatively treated AML $(p=0.002$, relative risk 5.38, 95\% confidence interval $1.75-16.49$ for RHAMM; $p=0.017$, relative risk 4.23 , 95\% confidence interval $1.29-13.78$ for failure to achieve complete remission). 
a

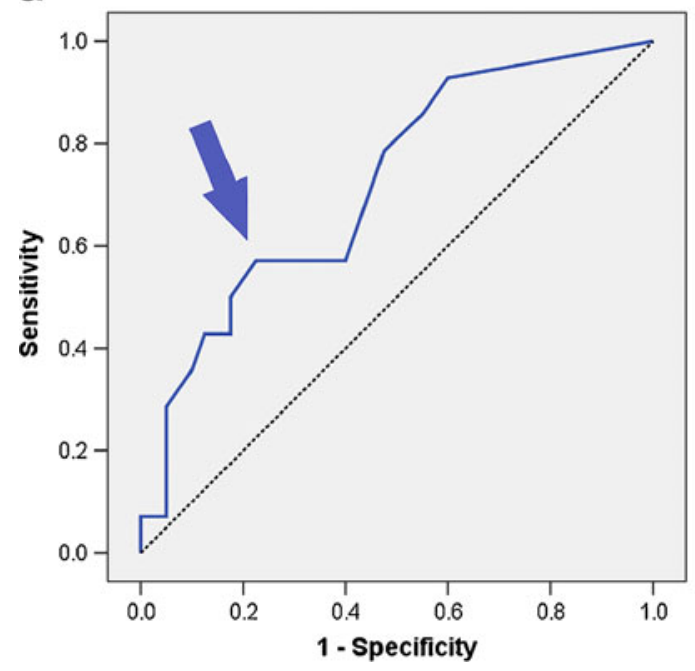

Fig. 2 Prognostic significance of RHAMM in AML. a ROC curve of the prognostic performance of RHAMM in AML cases treated with curative intent. The area under the ROC of $0.721(95 \%$ confidence interval $0.568-0.873$ ) indicates that consideration of RHAMM is $22 \%$ more accurate in predicting survival than a random guess $(p=0.015)$. The curve point (arrow) at sensitivity of 0.57 $(57 \%)$ and specificity of $0.775(77.5 \% ; 1$-specificity $=0.225)$ is at

\section{Discussion}

Our study is the first histopathology-based qualitative and quantitative examination of the expression of RHAMM and its partners in AML patients by immunohistochemistry on trephine bone marrow biopsies. Expression of RHAMM in $>5 \%$ of leukemic blasts identified a subgroup of AML patients (treated curatively) with poor prognoses independent of AML WHO subtype or age. This effect was b

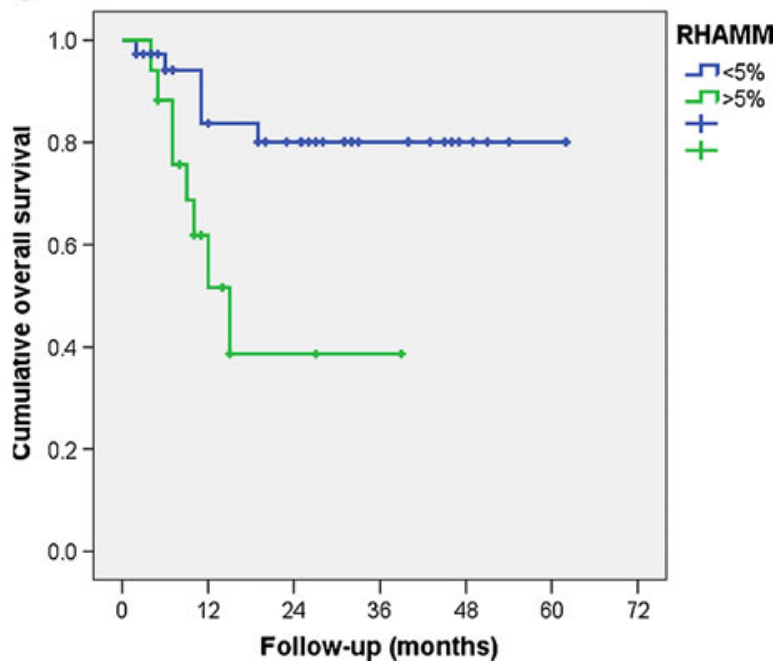

greatest distance from the reference line. The relevant cut-off score of RHAMM at that point is $>5 \%$ positive blasts. b Kaplan-Meier overall survival curves in AML patients treated with curative intent with respect to the expression of RHAMM; six out of the 37 RHAMM-negative AML patients died, mean survival 52 months, median not reached, compared to eight out of 17 RHAMM-positive patients, mean survival 21 months, median 15 months, $p=0.006$

particularly pronounced in patients who failed to achieve complete remission after induction therapy. Thus, our results considerably extend previous data on RHAMM in AML, showing that RHAMM not only represents a promising leukemia-associated antigen, similar to the preferentially expressed antigen in melanoma and the carbonic anhydrase IX (G250), with specific T-cell responses that suggest the possibility of tumor vaccination $[11,13]$, but also a prognostic tumor-related factor with a

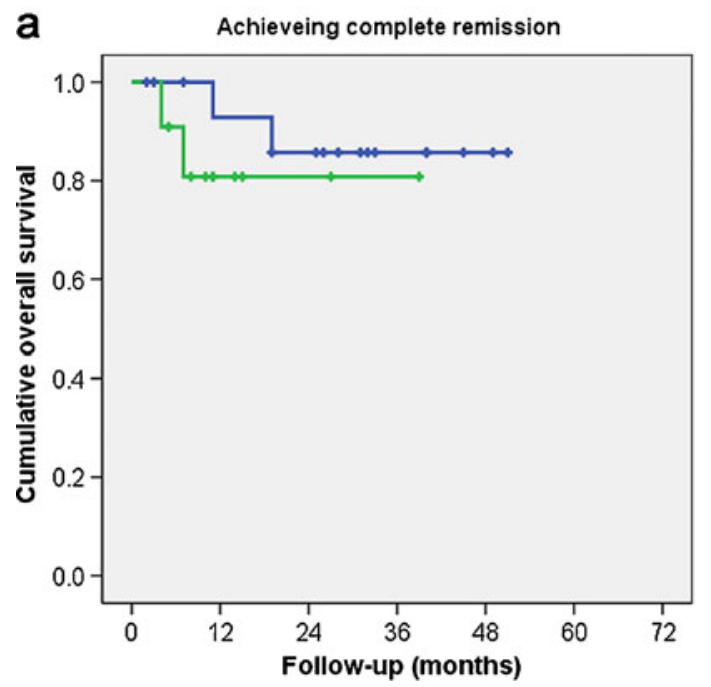

Fig. 3 Prognostic significance of RHAMM in AML with respect to treatment responses. Kaplan-Meier overall survival curves in AML patients achieving (a) and failing to achieve complete remission (b) with respect to the expression of RHAMM; $p=0.391$ for (a);

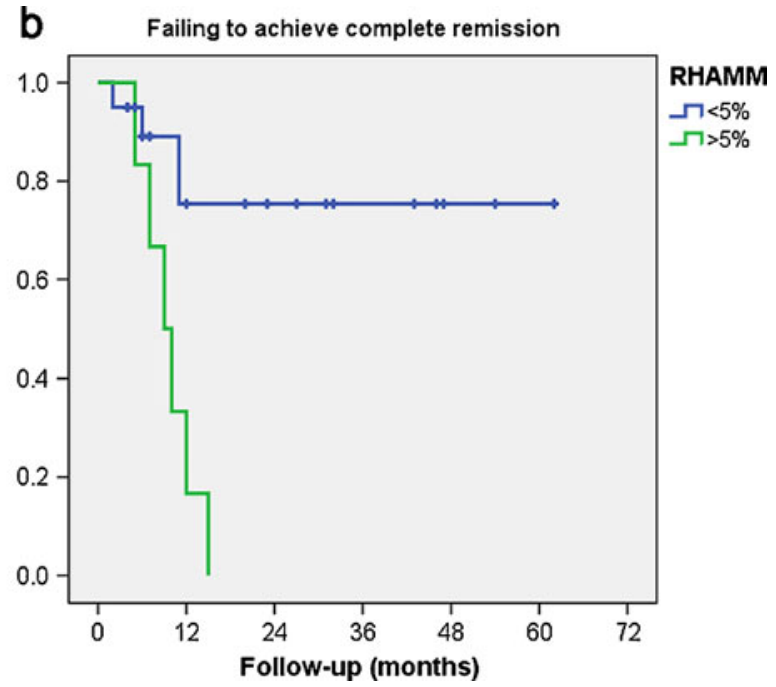

among the patients who failed to achieve complete remission four out of 20 RHAMM-negative patients died, mean survival 47 months, median not reached, compared to all six RHAMM-positive patients, mean survival 10 months, median 9 months, $p=0.001$ for (b) 
particular prognostic significance for patients failing to achieve complete remission after induction treatment. The latter might be of special interest since there are no established prognostic factors for AML in patients who failed to achieve complete remission.

RHAMM expression has been shown to be of prognostic importance in hematological neoplasia like B-cell chronic lymphocytic leukemia, plasma cell myeloma, and diffuse large B-cell lymphoma [10, 27-29]. The biological background of the observed prognostic effects of RHAMM is not known. Because it is, on the one hand, a surface receptor molecule, and on the other, a functional intracellular protein, RHAMM plays different roles that vary with respect to its numerous interaction partners. Together with CD44, RHAMM promotes cellular motility, wound healing, and the Ras-signaling cascade [15-17]. Through interactions with signaling related to ERK1/2, BRCA1 and BARD1, RHAMM, alone or in complexes with CD44, OPN, and integrins, RHAMM can be tumorigenic, most likely by inhibition of apoptosis [13-15, 18-23]. In addition, intracellular RHAMM helps maintaining genomic stability [21, 24]. Finally, as a surface molecule on tumor cells, RHAMM has been shown to play an important role in humoral anti-tumor responses [11, 25].

A former study of Greiner et al. suggest a possible yet not statistically significant $(p=0.284)$ favorable prognostic impact of RHAMM in AML [7]. These authors studied gene expression levels of tumor-associated antigens from peripheral blood and bone marrow samples utilizing mRNA quantification of extracts from frozen mononuclear cells. The probable positive prognostic effect of RHAMM was attributed to anti-leukemic activity of the immune system represented by killing residual tumor cells expressing such tumor-associated antigens. The discrepancy in the observed prognostic impact of RHAMM between our study and that of Greiner et al. might be due to the different methodologies employed to determine RHAMM expression, i.e., mRNA quantification and in situ analysis of blast counts with surface/cytoplasmic protein expression. This could reflect different RHAMM functions as an oncogene (surface, cytoplasmic protein), a tumor-suppressor gene (nuclear protein) or leukemia-associated antigen (surface and extracellular protein). Lastly, considering the controversies on the prognostic impact of expression levels of another leukemia-associated antigen, WT1, in the study of Greiner et al. and previous studies [7, 45, 46], and the minor, if any, statistical significance of the gene expression of all studied leukemia-associated antigens in the former study, the in situ approach chosen in our study might more ostensibly mirror the oncogenic function of RHAMM in AML. In our AML collective, RHAMM was predominantly cytoplasmically expressed in a proportion of blasts, and closely correlated with expression of CD44 and pSTAT3 and pSTAT5 as well as with spontaneous leukemia apoptosis, but did not correlate with OPN. This indicates that RHAMM is probably not constitutively expressed by all blasts, and that its observed effects might be related to the interference of OPN-independent RHAMM with AML cell signaling or locomotion, and rather not with its antiapoptotic functions.

In summary, beyond being a promising leukemiaassociated antigen in AML, consideration of RHAMM surface/cytoplasmic expression in blasts may allow identification of a subgroup of curatively treated patients with poor prognosis independent of AML WHO subtype, especially in patients who failed to achieve complete remission after induction therapy, which might merit further evaluation.

Acknowledgment The study was supported by the Oncosuisse grant OCS-01792-10-2005.

\section{References}

1. Estey E, Dohner H (2006) Acute myeloid leukaemia. Lancet 368:1894-1907

2. Löwenberg B (2008) Diagnosis and prognosis in acute myeloid leukemia - the art of distinction. N Engl J Med 358:1960-1962

3. Santamaria CM, Chillon MC, Garcia-Sanz R, Perez C, Caballero MD, Ramos F et al (2009) Molecular stratification model for prognosis in cytogenetically normal acute myeloid leukemia. Blood 114:148-152

4. Swerdlow S, Campo E, Harris N, Jaffe E, Pileri S, Stein H, Thiele J, Vardiman J (2008) WHO classification of tumours of haematopoietic and lymphoid tissues. IARC, Lyon

5. Wahlin A, Billstrom R, Bjor O, Ahlgren T, Hedenus M, Hoglund $M$ et al (2009) Results of risk-adapted therapy in acute myeloid leukaemia. A long-term population-based follow-up study. Eur J Haematol 83:99-107

6. Greiner J, Bullinger L, Guinn BA, Dohner H, Schmitt M (2008) Leukemia-associated antigens are critical for the proliferation of acute myeloid leukemia cells. Clin Cancer Res 14:7161-7166

7. Greiner J, Schmitt M, Li L, Giannopoulos K, Bosch K, Schmitt A et al (2006) Expression of tumor-associated antigens in acute myeloid leukemia: implications for specific immunotherapeutic approaches. Blood 108:4109-4117

8. Li L, Reinhardt P, Schmitt A, Barth TF, Greiner J, Ringhoffer M et al (2005) Dendritic cells generated from acute myeloid leukemia (AML) blasts maintain the expression of immunogenic leukemia associated antigens. Cancer Immunol Immunother 54:685-693

9. Giannopoulos K, Li L, Bojarska-Junak A, Rolinski J, Dmoszynska A, Hus I et al (2006) Expression of RHAMM/CD168 and other tumorassociated antigens in patients with B-cell chronic lymphocytic leukemia. Int J Oncol 29:95-103

10. Giannopoulos K, Mertens D, Buhler A, Barth TF, Idler I, Moller P et al (2009) The candidate immunotherapeutical target, the receptor for hyaluronic acid-mediated motility, is associated with proliferation and shows prognostic value in B-cell chronic lymphocytic leukemia. Leukemia 23:519-527

11. Greiner J, Ringhoffer M, Taniguchi M, Hauser T, Schmitt A, Dohner H, Schmitt M (2003) Characterization of several leukemia-associated antigens inducing humoral immune responses in acute and chronic myeloid leukemia. Int J Cancer 106:224-231 
12. Greiner J, Ringhoffer M, Taniguchi M, Li L, Schmitt A, Shiku H, Dohner H, Schmitt M (2004) mRNA expression of leukemiaassociated antigens in patients with acute myeloid leukemia for the development of specific immunotherapies. Int $\mathrm{J}$ Cancer 108:704-711

13. Greiner J, Ringhoffer M, Taniguchi M, Schmitt A, Kirchner D, Krahn $G$ et al (2002) Receptor for hyaluronan acid-mediated motility (RHAMM) is a new immunogenic leukemia-associated antigen in acute and chronic myeloid leukemia. Exp Hematol 30:1029-1035

14. Sherman L, Sleeman J, Herrlich P, Ponta H (1994) Hyaluronate receptors: key players in growth, differentiation, migration and tumor progression. Curr Opin Cell Biol 6:726-733

15. Tölg C, Hamilton SR, Nakrieko KA, Kooshesh F, Walton P, McCarthy JB, Bissell MJ, Turley EA (2006) Rhamm-/- fibroblasts are defective in CD44-mediated ERK1, 2 motogenic signaling, leading to defective skin wound repair. J Cell Biol 175:1017-1028

16. Turley EA, Belch AJ, Poppema S, Pilarski LM (1993) Expression and function of a receptor for hyaluronan-mediated motility on normal and malignant B lymphocytes. Blood 81:446-453

17. Wang C, Thor AD, Moore DH 2nd, Zhao Y, Kerschmann R, Stern R, Watson PH, Turley EA (1998) The overexpression of RHAMM, a hyaluronan-binding protein that regulates ras signaling, correlates with overexpression of mitogen-activated protein kinase and is a significant parameter in breast cancer progression. Clin Cancer Res 4:567-576

18. Hamilton SR, Fard SF, Paiwand FF, Tölg C, Veiseh M, Wang C et al (2007) The hyaluronan receptors CD44 and Rhamm (CD168) form complexes with ERK1, 2 that sustain high basal motility in breast cancer cells. J Biol Chem 282:16667-16680

19. Maxwell CA, McCarthy J, Turley E (2008) Cell-surface and mitoticspindle RHAMM: moonlighting or dual oncogenic functions? J Cell Sci 121:925-932

20. Bourguignon LY, Gunja-Smith Z, Iida N, Zhu HB, Young LJ, Muller WJ, Cardiff RD (1998) CD44v(3, 8-10) is involved in cytoskeleton-mediated tumor cell migration and matrix metalloproteinase (MMP-9) association in metastatic breast cancer cells. J Cell Physiol 176:206-215

21. Godar S, Weinberg RA (2008) Filling the mosaic of p53 actions: p53 represses RHAMM expression. Cell Cycle 7:3479

22. Khan SA, Cook AC, Kappil M, Günthert U, Chambers AF, Tuck AB, Denhardt DT (2005) Enhanced cell surface CD44 variant (v6, v9) expression by osteopontin in breast cancer epithelial cells facilitates tumor cell migration: novel post-transcriptional, posttranslational regulation. Clin Exp Metastasis 22:663-673

23. Tzircotis G, Thorne RF, Isacke CM (2005) Chemotaxis towards hyaluronan is dependent on CD44 expression and modulated by cell type variation in CD44-hyaluronan binding. J Cell Sci 118:5119-5128

24. Sohr S, Engeland K (2008) RHAMM is differentially expressed in the cell cycle and downregulated by the tumor suppressor p53. Cell Cycle 7:3448-3460

25. Schmitt A, Barth TF, Beyer E, Borchert F, Rojewski M, Chen J et al (2009) The tumor antigens RHAMM and G250/CAIX are expressed in head and neck squamous cell carcinomas and elicit specific CD8+ T cell responses. Int J Oncol 34:629-639

26. Gust KM, Hofer MD, Perner SR, Kim R, Chinnaiyan AM, Varambally S et al (2009) RHAMM (CD168) is overexpressed at the protein level and may constitute an immunogenic antigen in advanced prostate cancer disease. Neoplasia 11:956-963

27. Maxwell CA, Rasmussen E, Zhan F, Keats JJ, Adamia S, Strachan $E$ et al (2004) RHAMM expression and isoform balance predict aggressive disease and poor survival in multiple myeloma. Blood 104:1151-1158

28. Nagel S, Hirschmann P, Dirnhofer S, Günthert U, Tzankov A (2010) Coexpression of CD44 variant isoforms and receptor for hyaluronic acid-mediated motility (RHAMM, CD168) is an International Prognostic Index and C-MYC gene statusindependent predictor of poor outcome in diffuse large B-cell lymphomas. Exp Hematol 38:38-45

29. Pilarski LM, Masellis-Smith A, Belch AR, Yang B, Savani RC, Turley EA (1994) RHAMM, a receptor for hyaluronan-mediated motility, on normal human lymphocytes, thymocytes and malignant $B$ cells: a mediator in B cell malignancy? Leuk Lymphoma 14:363-374

30. Rein DT, Roehrig K, Schondorf T, Lazar A, Fleisch M, Niederacher D, Bender HG, Dall P (2003) Expression of the hyaluronan receptor RHAMM in endometrial carcinomas suggests a role in tumour progression and metastasis. J Cancer Res Clin Oncol 129:161-164

31. Shigeishi H, Fujimoto S, Hiraoka M, Ono S, Taki M, Ohta K, Higashikawa K, Kamata N (2009) Overexpression of the receptor for hyaluronan-mediated motility, correlates with expression of microtubule-associated protein in human oral squamous cell carcinomas. Int J Oncol 34:1565-1571

32. Zlobec I, Terracciano L, Tornillo L, Günthert U, Vuong T, Jass JR, Lugli A (2008) Role of RHAMM within the hierarchy of wellestablished prognostic factors in colorectal cancer. Gut 57:1413-1419

33. Mielgo A, van Driel M, Bloem A, Landmann L, Günthert U (2006) A novel antiapoptotic mechanism based on interference of Fas signaling by CD44 variant isoforms. Cell Death Differ 13:465-477

34. Ponta H, Sherman L, Herrlich PA (2003) CD44: from adhesion molecules to signalling regulators. Nat Rev Mol Cell Biol 4:33-45

35. Jin L, Hope KJ, Zhai Q, Smadja-Joffe F, Dick JE (2006) Targeting of CD44 eradicates human acute myeloid leukemic stem cells. Nat Med 12:1167-1174

36. Cheson BD, Bennett JM, Kopecky KJ, Buchner T, Willman CL, Estey EH et al (2003) Revised recommendations of the international working group for diagnosis, standardization of response criteria, treatment outcomes, and reporting standards for therapeutic trials in acute myeloid leukemia. J Clin Oncol 21:4642-4649

37. Schreck RR, Distèche CM (2001) Chromosome banding techniques. In: Haines JL, Korf BR, Morton CC, Seidman EG, Seidman JG, Smith DR, Sharer JD (eds) Current protocols in human genetics. Wiley, New York, pp 4.2.1-4.2.36

38. Grimwade D, Walker H, Harrison G, Oliver F, Chatters S, Harrison CJ et al (2001) The predictive value of hierarchical cytogenetic classification in older adults with acute myeloid leukemia (AML): analysis of 1065 patients entered into the United Kingdom Medical Research Council AML11 trial. Blood 98:1312-1320

39. Obermann EC, Arber C, Jotterand M, Tichelli A, Hirschmann P, Tzankov A (2010) Expression of pSTAT5 predicts FLT3 internal tandem duplications in acute myeloid leukemia. Ann Hematol 89:663-669

40. Atkins D, Reiffen KA, Tegtmeier CL, Winther H, Bonato MS, Storkel S (2004) Immunohistochemical detection of EGFR in paraffin-embedded tumor tissues: variation in staining intensity due to choice of fixative and storage time of tissue sections. J Histochem Cytochem 52:893-901

41. Zlobec I, Terracciano L, Jass JR, Lugli A (2007) Value of staining intensity in the interpretation of immunohistochemistry for tumor markers in colorectal cancer. Virchows Arch 451:763-769

42. Tzankov A, Zlobec I, Went P, Robl H, Hoeller S, Dirnhofer S (2010) Prognostic immunophenotypic biomarker studies in diffuse large B cell lymphoma with special emphasis on rational determination of cut-off scores. Leuk Lymphoma 51:199-212

43. Chen K, Liu J, Heck S, Chasis JA, An X, Mohandas N (2009) Resolving the distinct stages in erythroid differentiation based on dynamic changes in membrane protein expression during erythropoiesis. Proc Natl Acad Sci U S A 106:17413-17418 
44. Pilarski LM, Pruski E, Wizniak J, Paine D, Seeberger K, Mant MJ, Brown CB, Belch AR (1999) Potential role for hyaluronan and the hyaluronan receptor RHAMM in mobilization and trafficking of hematopoietic progenitor cells. Blood 93:2918-2927

45. Bergmann L, Miething C, Maurer U, Brieger J, Karakas T, Weidmann E, Hoelzer D (1997) High levels of Wilms' tumor gene (wt1) mRNA in acute myeloid leukemias are associated with a worse long-term outcome. Blood 90:1217-1225

46. Yanada M, Terakura S, Yokozawa T, Yamamoto K, Kiyoi H, Emi $\mathrm{N}$ et al (2004) Multiplex real-time RT-PCR for prospective evaluation of WT1 and fusion gene transcripts in newly diagnosed de novo acute myeloid leukemia. Leuk Lymphoma 45:1803-1808 